\title{
Under-reporting of dietary energy intake in five populations of the African diaspora
}

\author{
Lindsay Orcholski ${ }^{1}$, Amy Luke ${ }^{2}$, Jacob Plange-Rhule ${ }^{3}$, Pascal Bovet ${ }^{4,5}$, Terrence E. Forrester ${ }^{6}$, \\ Estelle V. Lambert ${ }^{7}$, Lara R. Dugas ${ }^{2}$, Elizabeth Kettmann ${ }^{2}$, Ramon A. Durazo-Arvizu ${ }^{2}$, \\ Richard S. Cooper ${ }^{2}$ and Dale A. Schoeller ${ }^{1 *}$ \\ ${ }^{1}$ Nutritional Sciences, University of Wisconsin, 425 Henry Mall, Madison, WI 53706, USA \\ ${ }^{2}$ Stritch School of Medicine, Loyola University Chicago, Maywood, IL, USA \\ ${ }^{3}$ Kwame Nkrumab University of Science and Technology, Kumasi, Ghana \\ ${ }^{4}$ Institute of Social and Preventive Medicine, Lausanne University Hospital, Lausanne, Switzerland \\ ${ }^{5}$ Ministry of Health, Republic of Seychelles \\ ${ }^{6}$ Tropical Medicine Research Institute, University of the West Indies, Mona, Kingston, Jamaica \\ ${ }^{7}$ Research Unit for Exercise Science and Sports Medicine, University of Cape Town, Cape Town, South Africa
}

(Submitted 26 November 2013 - Final revision received 22 October 2014 - Accepted 12 November 2014 - First published online 13 January 2015)

\begin{abstract}
Studies on the role of diet in the development of chronic diseases often rely on self-report surveys of dietary intake. Unfortunately, many validity studies have demonstrated that self-reported dietary intake is subject to systematic under-reporting, although the vast majority of such studies have been conducted in industrialised countries. The aim of the present study was to investigate whether or not systematic reporting error exists among the individuals of African ancestry ( $n$ 324) in five countries distributed across the Human Development Index (HDI) scale, a UN statistic devised to rank countries on non-income factors plus economic indicators. Using two $24 \mathrm{~h}$ dietary recalls to assess energy intake and the doubly labelled water method to assess total energy expenditure, we calculated the difference between these two values ((self-report - expenditure/expenditure) $\times 100)$ to identify under-reporting of habitual energy intake in selected communities in Ghana, South Africa, Seychelles, Jamaica and the USA. Under-reporting of habitual energy intake was observed in all the five countries. The South African cohort exhibited the highest mean under-reporting $(-52 \cdot 1 \%$ of energy) compared with the cohorts of Ghana $(-22.5 \%)$, Jamaica $(-17.9 \%)$, Seychelles $(-25.0 \%)$ and the USA $(-18.5 \%)$. BMI was the most consistent predictor of underreporting compared with other predictors. In conclusion, there is substantial under-reporting of dietary energy intake in populations across the whole range of the HDI, and this systematic reporting error increases according to the BMI of an individual.
\end{abstract}

\section{Key words: Diet assessments: $24 \mathrm{~h}$ recalls: Doubly labelled water: Human Development Index}

Energy imbalance, manifested as either underweight or overweight, is implicated in countless health problems and can lead to premature death. While undernutrition has long been the major health risk in developing countries, the contemporary primary concern is now overnutrition, which often exists contemporaneously with undernutrition. This shift in focus has been driven by the rapid increases in the prevalence of obesity across virtually every segment of nearly all populations around the world. According to the WHO, obesity (BMI $>30 \mathrm{~kg} / \mathrm{m}^{2}$ ) is increasing globally with many countries having rates greater than $10 \%$ and a few, particularly among Pacific Island nations, having rates in excess of $50 \%{ }^{(1)}$. Not surprisingly, public health investigators have undertaken numerous studies to understand the causes of the increase in the rates of obesity. Many of these studies have focused on potential dietary causes including energy intake that is collected using self-reported dietary instruments; however, self-reports are subject to both random error, which, by averaging, can still produce accurate results, and systematic error, which will introduce bias that may lead to erroneous conclusions regarding both the absolute amounts of foods consumed and the relationship between energy intake and nutritionally related diseases ${ }^{(2,3)}$.

Many studies have been conducted in industrialised countries to assess the validity of self-reported dietary intake across different age groups and ethnicities. In general, these studies have verified the finding of systematic under-reporting in self-reported dietary intake assessments with increasing bias

Abbreviations: DLW, doubly labelled water; HDI, Human Development Index; METS, Modelling the Epidemiologic Transition Study; NDSR, Nutrient Data System for Research; TEE, total energy expenditure; TEI, total energy intake.

* Corresponding author: D. A. Schoeller, fax +1 6082625860 , email dschoell@nutrisci.wisc.edu 
as BMI increases ${ }^{(4)}$. However, relatively few studies have been conducted in developing countries, and a large fraction of those have relied on indirect methods such as the comparison of self-reported energy intake with physiologically plausible energy requirements, calculated as multiples of $\mathrm{RMR}^{(5,6)}$. A more accurate assessment of under-reporting can be obtained using the biomarker approach of measured energy expenditure based on the doubly labelled water (DLW) $\operatorname{method}^{(7)}$.

Assessment of under-reporting by comparison of selfreported energy intake with actual total energy expenditure (TEE) is a corollary of the first law of thermodynamics: energy may neither be created nor destroyed. Metabolisable energy intake must equal TEE plus change in body energy stores. In weight-stable adults, change in body energy stores is small and, therefore, energy intake nearly equals TEE. Habitual energy intake, i.e. an individual's typical energy intake that maintains weight, is, therefore, taken to equal TEE. Actual energy intake may differ from habitual energy intake under certain conditions such as weight loss, where the reporting of energy intake can differ from habitual energy intake due to a reporting error or due to energy restriction during the reporting period ${ }^{(8)}$. However, both causes result in a systematic error in the assessment of habitual energy intake. The Modelling the Epidemiologic Transition Study (METS) included DLW measures and provides an opportunity to study under-reporting in community-based cohorts from five countries at differing stages of social and economic development as categorised by the Human Development Index (HDI). Participation in the METS provided us with an opportunity to assess the accuracy of self-reported dietary energy intake across the spectrum of the HDI and thus contribute to filling the gap in the knowledge of systematic reporting errors in developing countries. We hypothesised that under-reporting of dietary energy intake would be observed in all the five countries, but that it would increase between sites with increasing HDI and between individuals with increasing BMI.

\section{Experimental methods}

\section{Participants}

A total of 2500 young adults (age 25-45 years, approximately $60 \%$ female) were recruited in 2010 for participation in the $\operatorname{METS}^{(9)}$. Of this total, a subsample of 375 ( $n 75$ per site) were randomly selected for participation in the DLW protocol. The five sites included communities in rural Ghana, urban South Africa, Seychelles, urban Jamaica and suburban USA. The communities were not randomly selected, and, thus, our sample should not be viewed as representatives of each country's entire population but rather as representations of the lifestyles, including diet and physical activity patterns, of the respective communities. Individuals with infectious diseases such as malaria, who were HIV-positive, and who were pregnant were excluded from the study. Each of the cohorts was of Black African ancestry and exhibited a wide range of mean BMI from an average of $24 \mathrm{~kg} / \mathrm{m}^{2}$ in rural Nkwantakese (Ghana) to $32 \mathrm{~kg} / \mathrm{m}^{2}$ in suburban Maywood,
IL (USA). The five countries studied were selected to be diverse with respect to economic and social development as defined by the UN HDI of $2010^{(10)}$. The USA has been defined as a very high-HDI country, Jamaica and the Seychelles as high-HDI countries, South Africa as a middle-HDI country, and Ghana as a low-middle-HDI country ${ }^{(10)}$

The specific sites have been described by Luke et al. ${ }^{(9)}$. Briefly, the site in Ghana was the rainforest village of Nkwantakese and is in the Afigya Sekyere District in the Ashanti Region. This village has a population of 16965 and is about $20 \mathrm{~km}$ from Kumasi, the second largest city in Ghana. Approximately $10 \%$ of the men in our Ghanaian cohort commute to Kumasi for work daily, while a large proportion of the women (40\%) work in Kumasi or nearby villages as traders. Approximately a quarter of both men and women engage in subsistence agricultural work. In South Africa, the town studied was Khayelitsha, the third largest township in South Africa. Located next to Cape Town, the township has a population of almost 500000 and an unemployment rate of $40 \%$. Unemployment among our cohort was much lower than the community average at about $10 \%$. A significant proportion of both men and women $(>70 \%)$ were engaged in routine manual or service occupations such as construction work or transport among men and trading among women. In Jamaica, the participants studied were from the capital and largest city, Kingston, which has a population of 651880. As in South Africa, many of the male participants (>30\%) were engaged in construction work with another $20 \%$ working as mechanics or craftsmen. Women were predominantly engaged in routine manual and service occupations. In the Seychelles, participants were from the main island (Mahé, $27 \mathrm{~km}$ in length, which contributes to $90 \%$ of the total population of the country) with a majority of them commuting for work to the capital, Victoria. The Seychelles has a population of approximately 87000 and the archipelago is located in the Indian Ocean, about $1600 \mathrm{~km}$ east of Kenya. Occupations for the Seychellois cohort varied considerably, especially for men, with a lower proportion of manual labour than any of the other sites ( $40 \% v$. 50-90\%). The study site in the USA was Maywood, IL with a population of 24903 . A suburb of Chicago, Maywood is a working class, predominantly African American community. Over $50 \%$ of the men were engaged in routine service occupations, while about $45 \%$ of women had clerical or service representative positions.

Participants at each site were recruited by local investigators, and recruiting strategies within each site were at the discretion of the lead investigator. In Ghana, door-to-door recruitment was used. Researchers in the Seychelles, as well as South Africa, used sex- and age-stratified random samples, which were obtained from the national census of each country. For Jamaica, a fixed point in each district was determined (e.g. north-west corner), and from there random sampling occurred by means of door-to-door recruitment. Recruiters in Maywood used a similar approach, in which all city blocks in the community were randomised and then door-to-door recruitment took place. The present study was conducted according to the guidelines laid down in the Declaration of Helsinki, and all procedures involving human 
subjects were approved by the Institutional Review Boards or Ethics Committees of each of the six institutions ${ }^{(9)}$. Written informed consent was obtained from all the participants.

\section{Protocol}

Participants reported to their site-specific field clinic in the morning after an overnight fast, and baseline measurements were performed. Measurements of TEE using the DLW method, dietary intake using a $24 \mathrm{~h}$ recall, and body weight and height were obtained from each participant, while information about age and years of education was obtained by interview. Body-weight measurement was repeated at the end of the DLW period, approximately $7 \mathrm{~d}$ later. One additional $24 \mathrm{~h}$ dietary recall was collected from each participant 6-9d after the first visit.

\section{Energy intake (24h dietary recall)}

To provide an estimate of habitual intake, two $24 \mathrm{~h}$ dietary recalls were obtained from each participant ${ }^{(11)}$. The recalls were performed by trained interviewers using a multiplepass method, as designed by the Medical Research Council of South Africa ${ }^{(12)}$, in which the participants reported specific foods and their amounts consumed. Recall interview scripts were translated into the local language, where appropriate. These scripts guided the recording of all foods consumed on the previous day, first by a quick list and then by a meal-bymeal listing, followed by probing for commonly unreported foods. The assignment of portion size was based on representative photos of small, medium and large portions of all foods and food mixtures commonly consumed locally, as well as on the use of representative spoons, cups, bowls and plates. Details on preparation methods were sought with particular emphasis on the cooking method and the type of oils used, if appropriate.

In an effort to maximise the consistency of the format of the interview across the field sites and thus minimise between-site methodological biases, interviewers were either trained dietitians or nutritionists and those who had attended dietary recall training at Loyola University Chicago ${ }^{(11)}$. Because there were differences in the specific foods commonly consumed across the five countries, the methodology allowed for these differences. For example, before the initiation of participant enrolment, a dietetic consultant visited all the sites to photograph all commonly observed local foods and food mixtures at varying portion sizes (half, typical and oneand-one-half), to obtain local recipes, identify local measuring tools, and identify foods that might be commonly unreported. The photographs with portion size estimates were based on a system developed and utilised in South Africa ${ }^{(12)}$. The Medical Research Council dietetic consultant also monitored the local field staff while conducting $24 \mathrm{~h}$ dietary recall interviews in an effort to increase the fidelity of the recall method. All recalls and their details were recorded on standardised paper forms that were structured to coincide with the order of the script.

The recalls were digitised and sent to the Coordinating Center at Loyola University Chicago where analysis of the data was done using the Nutrient Data System for Research (NDSR; University of Minnesota, Minneapolis, MN) ${ }^{(9)}$ by the study dietitian. All recalls were assessed in an on-going manner by the study dietitian at Loyola, with requests for clarification relayed back to the sites immediately. Energy and nutrient contents of mixed food dishes from each of the sites that were not already part of the NDSR database were calculated from regional sources, e.g. West African, East African, South African or Caribbean nutrient databases ${ }^{(13-17)}$. These data were then used to identify comparable foodstuffs in the NDSR database. The lead study dietitian from Loyola travelled to the Ghanaian and Seychellois sites approximately 6 months after the initiation of participant enrolment to clarify any questions that arose during coding with regard to local ingredients and recipes. Issues that were not resolved during this visit with regard to the Jamaican and South African sites were settled via email and telephone discussion.

For the present analysis, energy intake was calculated for each food and quantity eaten and then totalled for each day. Total energy intake (TEI) was calculated as the mean of energy intake calculated from the two $24 \mathrm{~h}$ dietary recalls and expressed as $\mathrm{kJ} / \mathrm{d}$.

\section{Total energy expenditure (doubly labelled water method)}

The DLW method was used to measure TEE in the participants. In this method, a dose of DLW is administered and concentrations of ${ }^{2} \mathrm{H}$ and ${ }^{18} \mathrm{O}$ were measured in subsequent time- and date-stamped samples of urine using MS. The total amount of $\mathrm{CO}_{2}$ produced by the body in the interval can be estimated from the difference in the elimination rates of ${ }^{2} \mathrm{H}$ and ${ }^{18} \mathrm{O}$ over time. The method has been described in detail elsewhere $^{(18)}$. DLW is the standard method for assessing TEE. Urine samples collected at each site were transferred in o-ring-sealed, freezer-safe, plastic vials and kept frozen at each site. These were periodically shipped to Loyola in chilled thermo-boxes and stored frozen until shipped to the central analysis laboratory at the University of Wisconsin. Laboratory estimates of TEE have been shown to be unbiased and have a 1-sigma precision of $7 \%$ when analysed along with laboratory water standards in each batch that had been calibrated against international stable isotopic water standards ${ }^{(4)}$.

A baseline sample of urine was collected, and then a loading dose of the isotopic water was ingested orally. Urine samples were then collected at 1,3 and $4 \mathrm{~h}$ after ingesting the loading dose. After $7 \mathrm{~d}$, participants returned to the study centres and provided two final urine samples at a $1 \mathrm{~h}$ interval. $\mathrm{CO}_{2}$ production in each individual was calculated from the elimination rates of ${ }^{2} \mathrm{H}$ and ${ }^{18} \mathrm{O}$, and TEE calculated using the modified Wier equation and the average food quotient for each site ${ }^{(2)}$. The results of the TEE analyses were then combined with reported TEI, and the proportion of under-reporting was assessed by the ratio TEI:TEE.

\section{Anthropometric measurements}

Height was measured to the nearest $0 \cdot 1 \mathrm{~cm}$ using a wallmounted standard stadiometer (Invicta Stadiometer; Invicta) 
with the participant in erect position without shoes and head held in the Frankfort plane ${ }^{(9)}$. Similarly, weight was measured to the nearest $0 \cdot 1 \mathrm{~kg}$ (Seca 770) with the participant in light clothing and without shoes. The same standard calibrated balance scale was used at each of the five sites ${ }^{(9)}$. BMI was calculated as weight $(\mathrm{kg}) /$ height $^{2}\left(\mathrm{~m}^{2}\right)$. Weight was also measured at the end of the $7 \mathrm{~d}$ DLW period to assess whether there was a measurable change in body energy stores as estimated from weight change over the week of DLW measurement. Weight change was tracked to permit the estimation of energy imbalance and thus to distinguish between undereating and under-reporting in cases where TEI was less than TEE. Undereating, i.e. negative energy balance, results in the loss of body energy stores and hence body weight, while weight stability indicates under-reporting of energy intake. Years of education and age were self-reported by each participant.

\section{Statistical analysis}

Self-reported TEI data were compared with objectively measured TEE data. The primary outcome variable was the ratio of TEI based on $24 \mathrm{~h}$ dietary recalls to TEE based on the DLW method (i.e. TEI:TEE). A value of 1.0 would indicate perfect reporting with no error. The percentage of misreporting of energy was calculated as follows:

$$
(\text { TEI }- \text { TEE) } / \text { TEE } \times 100 .
$$

Here, a value of 0 would indicate no difference between the reported and measured values.

Data from the US site were used as the reference against which data from each of the other four countries were compared, as Maywood is a suburban, industrialised location

Table 1. Characteristics of the study participant by sex and site* and represents a community that we considered similar in nature to many previous studies of under-reporting in industrialised countries. Associations between the degree of under-reporting and BMI, sex, age and/or years of formal education were investigated, first in univariate models and then in a single multivariate model. BMI was examined as both a continuous variable in the models and a categorical value. The degree of under-reporting was calculated using standard BMI categories: underweight (BMI $<18.5 \mathrm{~kg} / \mathrm{m}^{2}$ ); healthy weight $\left(18.5-24.9 \mathrm{~kg} / \mathrm{m}^{2}\right)$; overweight $\left(25.0-29.9 \mathrm{~kg} / \mathrm{m}^{2}\right)$; obese $\left(\geq 30 \cdot 0 \mathrm{~kg} / \mathrm{m}^{2}\right)$. Likewise, years of education were included as a continuous variable in the models and by site-specific tertiles.

Self-reported energy intake was also assessed for physiological plausibility, i.e. too low to meet requirements for RMR and minimal physical activity. The standard chosen was the Goldberg threshold, which is based on estimated RMR. Dietary reports in which self-reported energy intake was $<1.35$ times the calculated RMR were considered as physiologically implausible outliers and, thus, deleted from the dataset. The RMR was calculated using the Mifflin-St Joer equation:

$$
\begin{aligned}
\text { RMR }= & 9.99 \times \text { weight }+6.25 \times \text { height }-4.92 \times \text { age }-166 \\
& \times \operatorname{sex}+5,
\end{aligned}
$$

where sex takes a value of 1 or 0 for female or male, respectively ${ }^{(6)}$.

\section{Results}

Of the 375 participants in the DLW protocol, data from 324 were used in these analyses. Those excluded from the dataset included twenty-one participants who had missing

\begin{tabular}{|c|c|c|c|c|c|c|c|c|c|c|c|c|c|c|c|}
\hline & \multirow[b]{2}{*}{$n$} & \multicolumn{2}{|c|}{ Age (years) } & \multicolumn{2}{|c|}{ Weight (kg) } & \multicolumn{2}{|c|}{ Height (cm) } & \multicolumn{2}{|c|}{ BMI $\left(\mathrm{kg} / \mathrm{m}^{2}\right)$} & \multicolumn{2}{|c|}{$\begin{array}{l}\text { Education } \\
\text { (years) }\end{array}$} & \multicolumn{2}{|c|}{ TEE (MJ/d) } & \multicolumn{2}{|c|}{ TEI (MJ/d) } \\
\hline & & Mean & SD & Mean & SD & Mean & SD & Mean & SD & Mean & SD & Mean & SD & Mean & SD \\
\hline \multicolumn{16}{|l|}{ Men } \\
\hline USA & 33 & 34 & 5 & 91 & 24 & 179 & 6 & 28 & 8 & 13 & 2 & $13 \cdot 0$ & 2.9 & $10 \cdot 1$ & 3.5 \\
\hline Seychelles & 35 & 34 & 5 & 73 & 11 & 170 & 6 & 25 & 4 & 12 & 2 & $12 \cdot 1$ & 1.9 & 8.4 & $2 \cdot 1$ \\
\hline Jamaica & 29 & 34 & 6 & 73 & 14 & 177 & 6 & 23 & 4 & 10 & 3 & $10 \cdot 6$ & 1.9 & 8.5 & $2 \cdot 4$ \\
\hline South Africa & 20 & 33 & 6 & 65 & 14 & 168 & 4 & 23 & 4 & 10 & 2 & $10 \cdot 0$ & $1 \cdot 8$ & 4.4 & 1.5 \\
\hline Ghana & 31 & 36 & 6 & 62 & 7 & 169 & 6 & 22 & 2 & 9 & 3 & $12 \cdot 1$ & 1.9 & 9.1 & 1.8 \\
\hline \multicolumn{16}{|l|}{ Women } \\
\hline USA & 30 & 35 & 6 & 91 & 17 & 164 & 5 & 34 & 7 & 13 & 2 & 9.8 & 1.5 & 7.9 & $2 \cdot 7$ \\
\hline Seychelles & 37 & 33 & 6 & 77 & 18 & 163 & 6 & 29 & 6 & 13 & 2 & $9 \cdot 3$ & 1.6 & $7 \cdot 1$ & $1 \cdot 8$ \\
\hline Jamaica & 34 & 35 & 6 & 76 & 15 & 164 & 6 & 28 & 6 & 11 & 2 & 8.7 & 1.3 & $7 \cdot 0$ & 1.7 \\
\hline South Africa & 39 & 34 & 6 & 84 & 25 & 162 & 7 & 32 & 9 & 10 & 2 & 9.7 & $1 \cdot 8$ & 4.4 & 1.2 \\
\hline Ghana & 36 & 35 & 6 & 64 & 16 & 158 & 5 & 26 & 7 & 6 & 4 & $10 \cdot 0$ & 1.9 & $7 \cdot 6$ & 1.6 \\
\hline \multicolumn{16}{|l|}{ Combined } \\
\hline USA & 63 & 34 & 6 & 91 & 21 & 172 & 9 & 31 & 8 & 13 & 2 & 11.5 & $2 \cdot 8$ & $9 \cdot 0$ & 3.3 \\
\hline Seychelles & 72 & 33 & 6 & 75 & 15 & 167 & 7 & 27 & 5 & 12 & 2 & $10 \cdot 7$ & $2 \cdot 2$ & 7.7 & $2 \cdot 0$ \\
\hline Jamaica & 63 & 34 & 6 & 75 & 15 & 170 & 9 & 26 & 5 & 11 & 3 & $9 \cdot 6$ & 1.9 & $7 \cdot 7$ & $2 \cdot 2$ \\
\hline South Africa & 59 & 34 & 6 & 78 & 24 & 164 & 7 & 29 & 9 & 10 & 2 & 9.8 & $1 \cdot 8$ & 4.4 & 1.3 \\
\hline Ghana & 67 & 35 & 6 & 63 & 13 & 163 & 8 & 24 & 6 & 7 & 4 & $10 \cdot 9$ & $2 \cdot 1$ & $8 \cdot 3$ & 1.9 \\
\hline Total & 324 & 34 & 6 & 76 & 20 & 167 & 9 & 27 & 7 & 11 & 3 & $10 \cdot 5$ & $2 \cdot 3$ & 7.5 & $2 \cdot 7$ \\
\hline
\end{tabular}
measurement data for TEE and thirteen participants who had

(Mean values and standard deviations)

TEE, total energy expenditure; TEI, total energy intake.

* Sites listed from the highest to the lowest Human Development Index ranking. 
Table 2. Average weight change* over the week of the doubly labelled water measurement period by sex and site $\dagger$

(Mean values and standard deviations)

\begin{tabular}{|c|c|c|c|c|}
\hline & \multicolumn{4}{|c|}{ Weight change $(\mathrm{kg})$} \\
\hline & \multicolumn{2}{|c|}{ Men } & \multicolumn{2}{|c|}{ Women } \\
\hline & Mean & SD & Mean & SD \\
\hline USA & 0.11 & 1.5 & 0.05 & 1.0 \\
\hline Seychelles & -0.01 & 0.9 & 0.17 & 0.7 \\
\hline Jamaica & -0.12 & 0.9 & 0.25 & $1 \cdot 1$ \\
\hline South Africa & 0.05 & $1 \cdot 2$ & 0.13 & 1.0 \\
\hline Total & 0.01 & 1.0 & 0.12 & 0.8 \\
\hline
\end{tabular}

*Weight change data were not available for Ghana.

† Sites listed from the highest to the lowest Human Development Index ranking

missing data on both $24 \mathrm{~h}$ dietary recalls. Data from seventeen participants were identified as outliers that had excessive numerical influence based on TEI:TEE ratios, i.e. more than 2 standard deviations from the mean, and were excluded from the dataset.

Participant characteristics are presented in Table 1. As expected, due to study design, body weight and BMI among men increased with increasing HDI ranking; the pattern was less consistent among women, with South Africans having a higher mean BMI than either Seychellois or Jamaicans but a lower HDI rank. Although TEI was significantly less than TEE in all the sites $(P<0 \cdot 001)$, among both men and women of South Africa, the difference was considerably larger than that in the other four sites. The intra-individual CV for selfreported TEI was significantly higher among South Africans $(16.9 \% ; P<0.01)$ than any of the other sites (USA $0.6 \%$, Seychelles $0.02 \%$, Jamaica $0.7 \%$ and Ghana $1.2 \%$; mean of all sites $2 \cdot 8 \%)$. With the exception of South Africa $(r-0.20)$, the correlation between TEI and TEE was consistent across the other four sites, ranging from $0 \cdot 21$ to $0 \cdot 28$ (mean of all sites $r$ 0.27).

Weight change over the week of the DLW measurement period was calculated, except in Ghana where weight data were not available for most subjects at the end of the assessment period. Weight changes over the $7 \mathrm{~d}$ of the DLW period averaged $0.25 \mathrm{~kg}$ or less in each subgroup, and none was significantly different from zero (Table 2). For the entire cohort, there was power to detect a weight change of $80 \mathrm{~g}$, which, assuming an energy equivalence of $31.0 \mathrm{~kJ} / \mathrm{g}$, corresponds to a change in energy stores of $2481 \mathrm{~kJ} /$ week or $356 \mathrm{~kJ} / \mathrm{d}$ from an average TEE of $10.51 \mathrm{MJ} / \mathrm{d}$. Thus, a difference between the self-reported TEI and TEE greater than $3.4 \%$ of TEE for the entire cohort or about $7 \%$ for an individual site represents a significant contribution from under-reporting.

The mean under-reporting expressed as a percentage of TEE $(((T E I-T E E) / T E E) \times 100)$ for all subjects combined was -27 (SD 26)\%, which was significantly different from zero $(P<0.001)$. Under-reporting was observed in all the five countries and for both sexes (Fig. 1). The mean percentages of under-reporting for all males and females were -26 (SD 26) \% and - 27 (SD 27) \%, respectively. Sex was not a significant predictor of under-reporting in the univariate model.
For the entire dataset, all BMI categories under-reported and the degree of under-reporting increased with increasing BMI. The mean percentages of energy under-reported for each BMI group were as follows: underweight -15.4 (SD 32.3 ) $\%$; healthy weight -22.3 (SD 23.8) \%; overweight -23.0 (SD 28.1) \%; obese -36.5 (SD 25.9) \% ( $P$ for trend <0.001). The distribution of under-reporting as a function of BMI by sex and site is shown in Fig. 2.

For the entire cohort, the degree of under-reporting did not differ by site-specific tertile of education $(P=0 \cdot 22)$; however, for both South African and Seychellois sites, there was less under-reporting as the level of education increased $(P<0.001$ and $P=0.03$, respectively). This was countered in the US site where under-reporting tended to increase with the tertile of education, moving from the lowest to the highest $(P=0.065)$. Thus, the influence of education was not consistent between the sites.

\section{Combined model}

When the predictors were combined in a final multivariate model, site, BMI and sex were found to be significant, but age and years of education, or tertiles of education, were not significant (Table 3). For every one-unit increase of BMI, under-reporting of TEI increased by $1.3 \%$; after controlling for the other covariates, including BMI, women underreported less than men by $9.6 \%$. Compared with the USA, under-reporting was significantly greater in Ghana by $12.6 \%$, the Seychelles by $12.4 \%$ and South Africa by $38.3 \%$ (all $P<0.02$ ), with the level of under-reporting in South Africa also being significantly greater than any of the other four countries $(P<0 \cdot 001)$. The significantly greater degree of under-reporting in the South African participants was identified as a cause for concern early in the study. To determine whether the method used by the Loyola dietitian, i.e. translation of local foods to foodstuffs available in the NDSR database, appropriately captured the energy content, the first set of two $24 \mathrm{~h}$ dietary recalls was sent back to the University of Cape Town and analysed by their on-staff, well-trained registered dietitian utilising the local nutrient database,

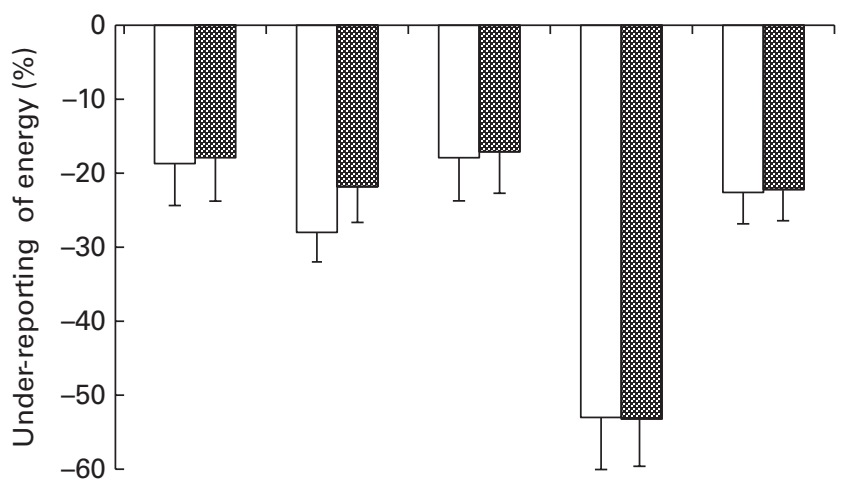

USA Seychelles Jamaica South Africa Ghana

Fig. 1. Under-reporting of energy intake as a percentage of measured total energy expenditure by site, listed from the highest to the lowest UN Human Development Index ranking. Values are means, with their standard errors represented by vertical bars. $\square$, Men; 囷, women. 


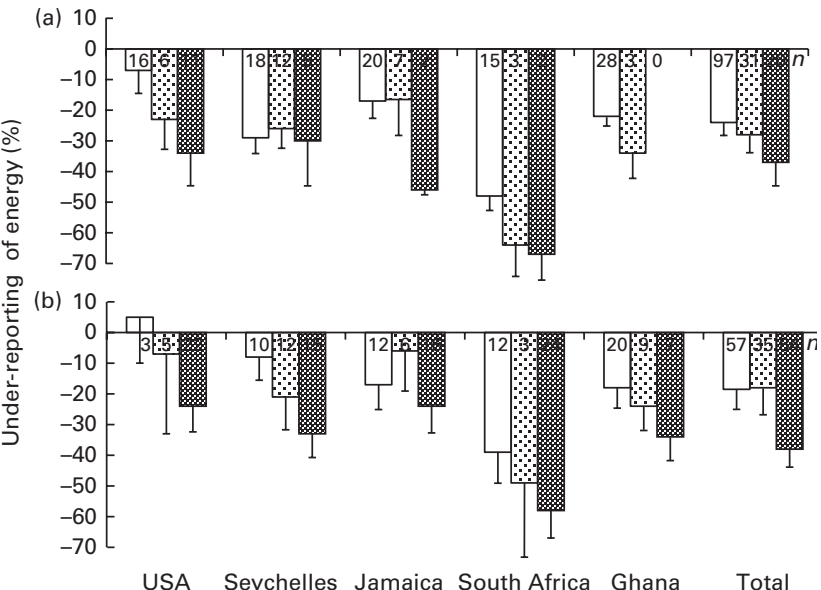

Fig. 2. Under-reporting of energy intake as a percentage of total energy expenditure as a function of BMI: (a) men and (b) women. Values are means, with their standard errors represented by vertical bars. The number on top of each bar represents the number of participants in each BMI group by site. $\square, \mathrm{BMI}<25 \mathrm{~kg} / \mathrm{m}^{2}$; ㅁ, BMI $25<30 \mathrm{~kg} / \mathrm{m}^{2}$; ⿴囗., $\mathrm{BMI}>30 \mathrm{~kg} / \mathrm{m}^{2}$.

FoodFinder ${ }^{\circledR}$ version 3 (National Nutritional Intervention Unit, Medical Research Council, Parow, Cape Town, South Africa, 2002). The mean difference value between Loyola and University of Cape Town was $-326 \mathrm{~kJ} / \mathrm{d}(-78 \mathrm{kcal} / \mathrm{d})$, and the correlation value between the sites was $0 \cdot 88$. Thus, it was decided that in order to maintain consistency across the sites, all analyses were continued to be conducted at Loyola.

The degree of under-reporting did not correlate with the economic development index as hypothesised. The rank correlation of under-reporting ratio $v$. the HDI was $f=0 \cdot 19$ $\left(r^{2}, \mathrm{NS}\right)$.

\section{Effect of the Goldberg cut-off}

The use of the Goldberg threshold to eliminate physiologically implausible self-reports of energy intake was investigated ${ }^{(19)}$. A large proportion ( $73 \%$ ) of the dietary reports fell outside the Goldberg threshold for dietary energy intake (Fig. 3). The greatest proportion of implausible self-reports was observed in South Africa, with $83 \%$ of participants failing the Goldberg test. As expected, the use of the Goldberg threshold, however, did reduce the mean value of under-reporting, but resulted in the dropout of the majority of dietary self-reports. The mean percentages of implausible self-reports identified by the Goldberg threshold were 57, 73, 75, 83 and $64 \%$ for the USA, Seychelles, Jamaica, South Africa and Ghana, respectively. When limiting the analysis to the sole reports identified as plausible by the Goldberg cut-offs, the values of self-reported energy intakes (TEI) were quite similar to those of TEE. The average underreporting relative to TEE in these remaining ninety-four participants was 2 (SD 22) \% (NS).

\section{Discussion}

The main conclusion drawn from the present study is that adults from five countries across a large spectrum of the HDI all tend to dramatically under-report dietary energy intake, and that these self-reports are, therefore, invalid estimates of true energy intake. Our findings extend the prior findings of under-reporting in high-HDI countries to those of under-reporting in middle- and low-middle-HDI countries. The variation in the magnitude of under-reporting between the five countries was not associated with HDI status as we had hypothesised. As expected from the findings of under-reporting in high-HDI countries, the difference was strongly associated with the participants' BMI. Our findings are the most accurate available to date because underreporting was evaluated by TEE that was assessed using the objective DLW criterion method.

We compared under-reporting in four of the countries against the USA because there have been multiple studies performed in the USA using the DLW method. The Observing Protein and Energy Nutrition (OPEN) study used a $24 \mathrm{~h}$ dietary recall and found the average TEI to be -18 and $-11 \%$ less than TEE for women and men, respectively, which is very similar to the TEI found among US women in the present study, but not as high as that found among our US men ${ }^{(20)}$. The OPEN population was older, had accumulated more years of formal education and was mostly white, thus differing in demographic characteristics from those of the US cohort in the present study. In another large adult study of underreporting assessed by a $24 \mathrm{~h}$ dietary recall, the results among women also are similar to the findings in our cohort $(-2004 v .-1766 \mathrm{~kJ} / \mathrm{d})$, but, again, men in our cohort had a larger degree of under-reporting of energy $(-2469 v$. $-356 \mathrm{~kJ} / \mathrm{d})^{(21)}$. Again, the participants in this study had different demographic characteristics than those in the present study, e.g. the former were slightly older and racial make-up was not provided. Finally, in one study that included over 118 participants of European ancestry and 115 participants of African ancestry, it has been found that those of African ancestry under-reported 3 percentage points more than those of European ancestry ${ }^{(22)}$. For comparison with the present study, the combined male/female cohort in the Arab et $a l .{ }^{(22)}$ study under-reported by $11 \%$ as determined using a computer-assisted $24 \mathrm{~h}$ dietary recall. Taken together, the results of our US cohorts are not dissimilar to those reported in other large cohorts; however, males in our cohort appear to under-report more than those observed in other cohorts.

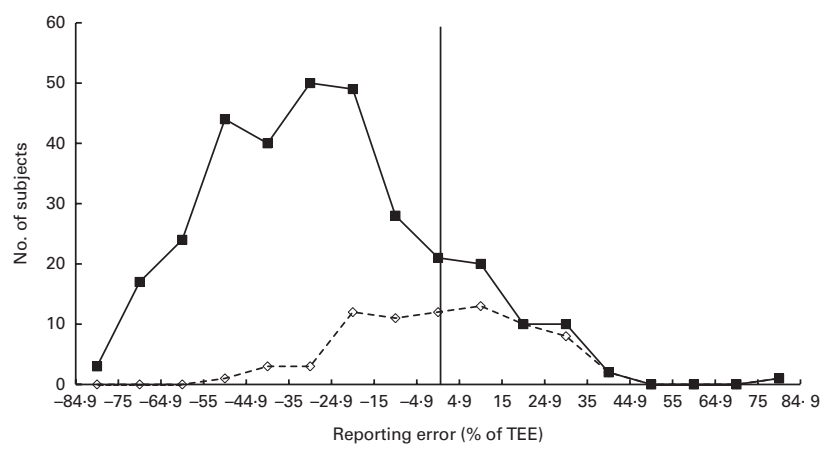

Fig. 3. Frequency distribution of under-reported energy intake as a percentage of measured total energy expenditure for the entire cohort $(-+)$ and for those who pass the Goldberg cut-off of $1.35 \times$ predicted resting energy expenditure $(-\diamond-)$. The vertical line represents $0 \%$ error. 
Table 3. Multivariate associations between the selected predictors and the percentage of under-reporting in individuals* ( $\beta$-Coefficients and standard errors)

\begin{tabular}{lrcc}
\hline & \multicolumn{1}{c}{$\beta$} & SE & $P$ \\
\hline BMI $\left(\mathrm{kg} / \mathrm{m}^{2}\right)$ & -1.3 & 0.2 & $<0.001$ \\
Sext & 9.6 & 2.9 & 0.001 \\
Age (years) & -0.1 & 0.2 & 0.77 \\
Education (tertilet) & 1.9 & 1.8 & 0.31 \\
Site $v$. USA & & & \\
$\quad$ Seychelles & -12.4 & 4.0 & 0.002 \\
Jamaica & -6.4 & 4.2 & 0.13 \\
South Africa & -38.3 & 4.2 & $<0.001$ \\
$\quad$ Ghana & -12.6 & 5.1 & 0.013 \\
Intercept & 6.2 & 10.9 & 0.57 \\
\hline
\end{tabular}

* Dependent variable in the regression model was the under-reporting of TEI as a percentage of TEE; a negative $\beta$-coefficient indicates an inverse association, i.e. as BMI increases, the TEI:TEE ratio decreases (indicating a higher percentage of under-reporting).

$\dagger$ Men $=0$ and women $=1$; site-specific tertiles of education: lowest $=1$ and highest $=3$.

The present study was not the first assessment of underreporting in countries of less than high HDI. In a review by Scagliusi et al. ${ }^{(5)}$, only two studies using the DLW method were identified and the third was reported later. A very small study from Swaziland has found no under-reporting, while the results from studies in Mexico and Brazil $(-21$ and $-17 \%$ of energy, respectively) are consistent with our findings in the Seychelles, Jamaica and Ghana. All studies were conducted among females. The study from Swaziland appears to be an outlier possibly due to the small number of participants ( $n$ 14) who were young (18-28 years) female students ${ }^{(23)}$. In the review by Scagliusi et al. ${ }^{(5)}$, nine studies from other developing countries relied on under-reporting assessed using the Goldberg criteria rather than TEE measured using the DLW method. Each study used a slightly different method of analysis; however, in each population, it was also concluded that dietary intake was under-reported ${ }^{(5)}$. To our knowledge, the only other multi-country study was conducted by Mennen et $a l .{ }^{(24)}$. This study was also performed in the participants of both sexes of African ancestry in urban Jamaica, rural and urban Cameroon, and the UK. The study used FFQ for measuring TEI and a calculated TEE based on the predicted RMR and a minimal physical activity factor to estimate under-reporting ${ }^{(24)}$. Women and men from Jamaica both under-reported significantly more than the participants from urban and rural Cameroon. Those from the UK had the highest under-reporting values, but were very similar to those from Jamaica ${ }^{(24)}$. Thus, although they have found under-reporting in all the countries, they have reported a strong relationship between increasing under-reporting and increasing economic development, which is in contrast to our findings. MacIntyre et al. ${ }^{(25)}$ also tested the accuracy of a FFQ in a population of South Africa, and made their assessment tool culturally specific by including traditional local foods. They have reported a much lower ratio of energy intake:calculated RMR than did Mennen et al. ${ }^{(24)}$ for the Cameroonians, suggesting a larger degree of underreporting, which, although less than what we observed in South Africans, was more similar to our general findings when we averaged across the countries. The percentage of under-reporters in these studies varied between 10 and $75 \%$ with a median of $22 \%$. In the present study, the percentage of under-reporters using the Goldberg cut-off was much higher and varied from $83 \%$ in South Africa to $57 \%$ in the USA. Our findings in the South African cohort were surprising compared with the report cited above, which found that only $43 \%$ of their cohort were classified as under-reporters by FFQ, while we employed trained interviewers and a multiple-pass recall, which in other studies has performed better than FFQ.

We found that BMI was, by far, the most consistent predictor of under-reporting. It was the strongest predictor and observed in each of the five countries. This is consistent with the studies conducted by Mennen et al. ${ }^{(24)}$, MacIntyre et $a l .{ }^{(25)}$ and Bedard et $a l .{ }^{(26)}$, as well as every study included in the review by Scagliusi et al. ${ }^{(5)}$. The consistency between our findings and the results of many other studies strengthens the conclusion that BMI is a major predictor of underreporting in populations of African descent regardless of the degree of social and economic development for the country, and it does so by providing a more quantitative measure.

However, the present results with respect to sex did not totally agree with previous studies. While a large number of men were classified as under-reporters, the difference between sexes in our multivariate model was smaller than that found by others, with the exception of the Seychelles ${ }^{(3,5)}$. Assessment of under-reporting, however, was not the primary aim of the METS, and thus we did not include measures of body image or social desirability that could help explain under-reporting patterns. Differences in body-weight ideals between men and women underlie the need for further studies to assess under-reporting according to BMI and sex in different socio-cultural contexts. The inclusion of this and other psychological measures would have been valuable to explain site-specific differences in under-reporting as well as a possible role or modifying effect of education ${ }^{(27)}$. In the USA, under-reporting only tended to increase with more education, but decreased with more education in South Africa and the Seychelles, while no association was observed in the other two countries. We suggest that further studies of dietary under-reporting include psychological and cultural measures, as well as indicators of political or social significance. For example, participants in some situations may fear losing food assistance if they report a large dietary intake or may feel a general distrust of authorities and purposely try to minimise information provided to authority figures.

To provide a comparison with several other studies, we applied the Goldberg cut-off method to our dataset in order to eliminate dietary reports in which the reported intake was so low relative to physiological energy requirements that it can be reasoned that the reported intake is too low to represent habitual energy intake ${ }^{(19)}$. After eliminating reported intakes that were $<1.35 \times$ REE, we did find that the average reported energy intakes did not differ from the energy expenditure biomarker of habitual energy intake. However, the use of the Goldberg cut-off had a significant cost with regard to the loss of data. This cut-off eliminated $73 \%$ of the intake 
records and raises concern over whether the remaining data are representative of all of the cohorts.

The severe under-reporting from the South African cohort was unexpected and cannot be explained based on the data that we collected. The TEE values used as the criterion method for estimating habitual energy intake fell on the same line, describing the relationship between TEE and fatfree mass, and fat-free mass was consistent with the BMI of the participants (data not shown). As indicated above, dietary coding performed at Loyola was double-checked and confirmed by a co-investigator from South Africa, who had adapted the recall methodology for use in the METS. As such, we do not believe that the discrepancy is due to methodological error. We are left to speculate that severe under-reporting by the South African cohort is due to a concern by participants over the loss of food assistance or some other factor that caused them to resist answering the dietary recall questions more accurately.

As indicated above, one of the limitations of this data analysis is the absence of psychological measures. A further limitation of the present study is that the participant samples were not representative of the country as a whole, and, therefore, the present results are more reflective of the community from which the data were taken. It is probable that there is more variation within each country than we have reported here. Also, virtually all subjects were of African ancestry by design and inference of our findings is thus limited to a single race, although to multiple countries of origin. The $24 \mathrm{~h}$ dietary recalls cover only a short time frame and were carried out mostly during weekdays, and thus may not be completely representative of the average for the participant had weekends been included. Seasonal effects could have skewed these measurements as well as personal situations such as injury or illness to prevent physical activity. In addition, different individuals collected the dietary data in each site, which could lead to biases between countries in our measurements.

Another limitation of the present study is that fifty-one participants in the study were not included in the final analysis. However, the number of lost cases was not unexpected. There were nineteen participants who did not have TEE values, which is close to the average of $4 \%$ for other studies. Participants without dietary recall data were $3 \%$ of the cohort, which does not seem to be excessive, and the $4 \%$ eliminated as outside $2 \mathrm{SD}$ of the country-specific mean with respect to reporting error was as expected based on statistics. It should also be noted that the characteristics of these participants were within $0.33 \mathrm{SD}$ units of the mean 324 subjects reported herein with respect to height, weight, age and years of education, and where data were available for dietary energy recall and TEE. The seventeen participants who were excluded as outliers did exhibit a trend away from underreporting, but their inclusion would have changed the mean under-reporting for the cohort from -27 to $-23 \%$, which would not have influenced the conclusions.

A major inference from the present study is that underreporting of dietary intake among adults appears to be an almost universal problem. Such under-reporting complicates the study of diet and health because when there is a lack of an accurate picture of what an individual is consuming on a regular basis, care should be taken when interpreting any study based on self-reported dietary data. Clearly, the present study and others invalidate the use of self-reported energy intake data for use in estimating energy balance until an accurate assessment method is identified. Also, because it is still unclear what components of the diet are under-reported, self-reported dietary pattern data must be interpreted with caution ${ }^{(28-30)}$. The present study found that under-reporting of dietary intake was strongly associated with BMI. Since social patterning of BMI may differ in lowand high-income countries, further studies should assess cross-cultural mechanisms underlying the association between BMI and under-reporting, e.g. body-weight ideals in different socio-cultural contexts. The present study and others further demonstrate the need for research directed towards the development of objective methods for the measurement of diet.

\section{Acknowledgements}

The authors thank the participants of the METS as well as the field staff in each of the study sites.

The present study was supported in part by the National Institutes of Health (DK080763).

The authors' contributions are as follows: A. L., J. P.-R., P. B., T. E. F., E. V. L., L. R. D., R. A. D.-A. and D. A. S. were instrumental in formulating the research questions in the METS project; A. L., J. P.-R., P. B., T. E. F., E. V. L., L. R. D., R. A. D.-A., R. S. C. and D. A. S. were involved in the study design; E. K. carried out the dietary assessments across all the sites; L. O. conducted the primary data reduction; L. O., A. L., L. R. D. and D. A. S. contributed to the data analysis; L. O., A. L., P. B., L. R. D. and D. A. S. contributed to the writing and editing of the final manuscript. All authors made a substantial contribution to the completion of the manuscript.

D. A. S. is a co-inventor on a patent application for a method to estimate energy balance.

\section{References}

1. World Health Organization (2006) Global database on body mass index. http://apps.who.int/bmi/ (accessed June 2014).

2. Trabulsi J \& Schoeller DA (2001) Evaluation of dietary assessment instruments against doubly labeled water, a biomarker of habitual energy intake. Am J Physiol Endcrinol Metab 281, E891-E899.

3. Subar AF, Kipnis V, Troiano RP, et al. (2003) Using intake biomarkers to evaluate the extent of dietary misreporting in a large sample of adults: the observing protein and energy nutrition (OPEN) study. Am J Epidemiol 158, 1-13.

4. Neuhouser M, Tinker L, Shaw PA, et al. (2008) Use of recovery biomarkers to calibrate nutrient consumption self-reports in the Women's health initiative. Am J Epidemiol 167, $1247-1259$.

5. Scagliusi FB, Ferriolli E \& Lancha AH Jr (2006) Underreporting of energy intake in developing nations. Nutr Rev $\mathbf{6 4}$, 319-330.

6. Frankenfield D, Roth-Yousey L \& Compher C (2005) Comparison of predictive equations for resting metabolic rate in 
healthy nonobese and obese adults: a systematic review. J Am Diet Assoc 105, 775-789.

7. Schoeller DA (2002) Validation of habitual energy intake. Public Health Nutr 5, 883-888.

8. Prentice AM, Black AE, Coward WA, et al. (1986) High levels of energy expenditure in obese women. Br Med J (Clin Res Ed) 292, 983-987.

9. Luke A, Bovet P, Forrester TE, et al. (2011) Protocol for the modeling the epidemiologic transition study: a longitudinal observational study of energy balance and change in body weight, diabetes and cardiovascular disease risk. $B M C$ Public Health 11, 927.

10. Barro RJ \& Lee JW (2010) A new data set of educational attainment in the world, 1950-2010. In The National Bureau of Economic Research. Cambridge, MA: National Bureau of Economic Research. http://www.nber.org/papers/ w15902 (accessed April 2011).

11. Gibson RS (1990) Methods for measuring food consumption of individuals. In The Principles of Nutritional Assessment, 2nd ed., pp. 41-43. New York, NY: Oxford University Press.

12. Steyn NP \& Senekal M (2005) A Guide for the Use of the Dietary Assessment and Education Kit (DAEK). Cape Town: Medical Research Council of South Africa.

13. Sharma S, Yacavone XC, Samuda PM, et al. (2009) Nutritional composition of commonly consumed composite dishes for Afro-Caribbeans (mainly Jamaicans) in the United Kingdom. Intl J Food Sci Nutr 60, 140-150.

14. US Department of Health Education and Welfare and Food Nutrition Program, Agricultural Organization of the United Nations (1968) Food Composition Table for Use in Africa. Bethesda, MD: Public Health Service.

15. The Caribbean and Nutrition Institute (1998) Food Composition Tables for Use in the English-speaking Caribbean. Vienna: World Health Organization.

16. Langenhovan ML, Conradle PJ, Wolmarans P, et al. (1991) MRC Food Quantities Manual, 2nd ed. Cape Town: Medical Research Council.

17. Sehmi JK (1993) National Food Composition Tables and the Planning of Satisfactory Diets in Kenya. Nairobi: Ministry of Health.

18. International Atomic Energy Agency (2009) Assessment of Body Composition and Total Energy Expenditure in Humans Using Stable Isotope Techniques. IAEA Human Health Series No. 3. Vienna: IAEA. http://www-pub.iaea. org/MTCD/publications/PDF/Pub1370_web.pdf (accessed December 2013).
19. Goldberg GR, Black AE, Jebb SA, et al. (1991) Critical evaluation of energy intake data using fundamental principles of energy physiology: 1 . Derivation of cut-off limits to identify under-recording. Eur J Clin Nutr 45, 569-581.

20. Tooze JA, Krebs-Smith SM, Troiano RP, et al. (2012) The accuracy of the Goldberg method for classifying misreporters of energy intake on a food frequency questionnaire and 24-h recalls: comparison with doubly labeled water. Eur J Clin Nutr 66, 569-576.

21. Novotny JA, Rumpler WV, Riddick H, et al. (2003) Personality characteristics as predictors of underreporting of energy intake on 24-hour dietary recall interviews. J Am Diet Assoc 103, 1146-1151.

22. Arab L, Tseng CH, Ang A, et al. (2011) Validity of a multipass, web-based, 24-hour self-administered recall for assessment of total energy intake in blacks and whites. Am J Epidemiol 174, 1256-1265.

23. Huss-Asmore R, Goodman JL, Sibiya TE, et al. (1989) Energy expenditure of young Swazi women as measured by the doubly labeled water method. Eur J Clin Nutr 43, 737-774.

24. Mennen LI, Jackson M \& Cade J (2000) Underreporting of energy intake in four populations of African origin. Int $J$ Obes Metab Disord 24, 882-887.

25. MacIntyre UE, Venter CS \& Vorster HH (2001) A culture-sensitive quantitative food frequency questionnaire used in an African population: 2. Relative validation by 7 -day weighted records and biomarkers. Public Health Nutr 4, 45-51.

26. Bedard D, Shatenstein B \& Nadon S (2004) Underreporting of energy intake from a self-administered food-frequency questionnaire completed by adults in Montreal. Public Health Nutr 7, 675-681.

27. Alwan H, Viswanathan B, Williams J, et al. (2010) Association between weight perception and socioeconomic status among adults in the Seychelles. BMC Public Health 10, 467.

28. Lafay L, Mennen L, Basdevant A, et al. (2000) Does energy intake underreporting involve all kinds of food or only specific food items? Results from the Fleurbaix Laventie Ville Santé (FLVS) study. Int J Obes Relat Metab Disord 24, 1500-1506.

29. Cagliusi FB, Ferriolli E, Pfrimer K, et al. (2010) Under-reporting of energy intake is more prevalent in a healthy dietary pattern cluster. BrJ Nutr 100, 1060-1068.

30. Suchanek P, Poledne R \& Hubacek JA (2011) Dietary intake reports fidelity - fact or fiction? Neuro Endocrinol Lett 32, Suppl. 2, 29-31. 